\title{
Genome-wide prioritization reveals novel gene signatures associated with cardiotoxic effects of tyrosine kinase inhibitors
}

\author{
YILAN LI ${ }^{1,2^{*}}$, WEIJIE WANG ${ }^{1,2^{*}}$, RONG GAO $^{1}$, XUEMING XU $^{1}$ and YAO ZHANG ${ }^{1,2}$ \\ ${ }^{1}$ Department of Cardiology, The 2nd Affiliated Hospital of Harbin Medical University; ${ }^{2}$ Key Laboratory of \\ Myocardial Ischemia, Ministry of Education, Harbin Medical University, Harbin, Heilongjiang 150000, P.R. China
}

Received March 13, 2020; Accepted October 30, 2020

DOI: 10.3892/ol.2020.12355

\begin{abstract}
Tyrosine kinase inhibitors (TKIs) are characterized as multi-targeted anticancer agents that lack specificity, leading to cardiovascular adverse effects. To date, there are no reliable means to predict the cardiotoxicity of TKIs under development. The present study assessed the usual variants of genes to determine the molecular targets of TKIs associated with heart failure (HF). Gene or gene products affected by TKIs were assessed using the Drug Gene Interaction Database. These genes were investigated in genome-wide association studies (GWAS) datasets associated with HF at a genome-wide significant level $\left(\mathrm{P}<1 \times 10^{-5}\right)$. Subsequently, single-nucleotide polymorphisms (SNPs) that reached the established GWAS threshold $\left(\mathrm{P}<5 \times 10^{-8}\right)$ were investigated for genome-wide significance. Based on a threshold score of 3 , nine gene loci yielded associations according to their biological function using RegulomeDB. Finally, comprehensive functional analysis of SNPs was performed using bioinformatics databases to identify potential drug targets. Using rSNPBase, rs7115242, rs143160639 and rs870064 were found to interfere with proximal transcription regulation, while rs7115242, rs143160639 and rs117153772 were involved in distal regulation, and most SNPs participated in post-transcriptional RNA binding protein-mediated regulation. rs191188930 on platelet-derived growth factor receptor (PDGFR) $\alpha$ was associated with numerous TKI drugs, including sunitinib, pazopanib, sorafenib, dasatinib and nilotinib. Using RegulomeDB and HaploReg v4.1, rs191188930 was predicted to be located in enhancer histone markers. PhenoScanner GWAS analysis revealed that rs191188930 was associated with other diseases
\end{abstract}

Correspondence to: Dr Yao Zhang, Department of Cardiology, The 2nd Affiliated Hospital of Harbin Medical University, 246 Xuefu Road, Nangang, Harbin, Heilongjiang 150000, P.R. China

E-mail: yaozhang_grace@163.com

${ }^{*}$ Contributed equally

Key words: tyrosine kinase inhibitors, heart failure, cardiotoxicity, platelet-derived growth factor receptor $\alpha$, Drug Gene Interaction Database or phenotypes, in addition to HF. Genotype-Tissue Expression analysis indicated that the PDGFR $\alpha$ gene had the highest median expression in 'Cells-Transformed fibroblasts', and the Search Tool for the Retrieval of Interacting Genes/Proteins revealed the protein-protein interaction network of PDGFR $\alpha$. The present findings demonstrated the overlap of TKI-induced genes and those mediating HF risk, suggesting molecular mechanisms potentially responsible for TKI-induced HF risk. Additionally, the present genetic study may be helpful to further investigate off-target drug effects.

\section{Introduction}

Left ventricular dysfunction and heart failure (HF), which are typically described as cardiotoxicity, are the most concerning cardiovascular complications of anticancer chemotherapies, which cause an increase in morbidity and mortality (1). Based on previous studies of anthracycline cardiotoxicity, increasing attention has focused on tyrosine kinase inhibitors (TKIs), a type of pharmaceutical drug inhibiting tyrosine kinases by competitively binding to and inhibiting their ATP binding pocket (2-5). TKIs are characterized by being multi-targeted anticancer agents that lack sufficient specificity, which markedly increases the risk of cardiotoxicity (5). In a prospective study of patients with metastatic renal-cell carcinoma, 3-15\% of patients developed cardiac dysfunction induced by the TKIs sunitinib, pazopanib and axitinib used after chemotherapy and $1-10 \%$ of patients showed symptomatic HF (1). Nevertheless, there are no reliable means to predict TKI-induced cardiotoxicity under development. As the cancer patient population ages, these potential cardiac adverse effects will become more prominent, and the need for improved prediction and prognosis will become even more pressing (6). Single nucleotide polymorphisms (SNPs) affecting disease risk can facilitate the identification of drug targets (7). Similarly, if risk alleles and drugs have equal functional implications, SNPs may point to cardiac complications.

The present study aimed to integrate genes or gene products interacting with TKIs in the Drug Gene Interaction Database (DGIdb). In order to prioritize and identify potential drug targets, the present study comprehensively investigated knownmoleculartargets of TKIsin HF-associated genome-wide association studies (GWAS) databases and identified common SNPs of these genes using multiple bioinformatics databases 
including PhenoScanner, RegulomeDB, HaploReg v4.1, rSNPBase, the University of California Santa Cruz (UCSC) Genome Browser and the Search Tool for the Retrieval of Interacting Genes/Proteins (STRING) database. The present study successfully prioritized SNPs and annotated the function of TKI-induced genes associated with HF, providing a foundation for further understanding of TKI-induced pathogenesis and the molecular mechanism of cardiotoxicity.

\section{Materials and methods}

DGIdb and GWAS datasets. Firstly, genes or gene products reported to interact with TKIs were identified in DGIdb v2.22 (8-10). DGIdb integrates existing resources to generate hypotheses on how mutant genes may be targeted for therapy, which provides an interface for a general search of genes for drug-gene interactions and potentially drug-available genes (8). Subsequently, respective genes in HF-associated GWAS datasets were screened from PhenoScanner (11). Over the last decade, GWAS datasets has facilitated our understanding of the potential role of genetics in variable responses to drugs and provided significant insight into genetic structure and the large number of SNPs associated with complex diseases (12). A total of $549 \mathrm{HF}$-associated SNPs was identified from previous GWAS with a P-value $<1 \times 10^{-5}$.

Regulatory analysis using RegulomeDB. SNPs interacting with TKIs for association with cardiotoxicity were searched further in RegulomeDB (13). RegulomeDB is a database including high-throughput experimental datasets from the Encyclopedia of DNA Elements (ENCODE) project, Gene Expression Omnibus and other sources, and is an important predictive tool to annotate and prioritize potential regulatory SNPs in the human genome, as well as to compute predictions and manual annotations to determine the assumed regulatory potential and identify functional variations (14). The known regulatory DNA elements include DNAase hypersensitivity regions, transcription factor binding sites and promoter regions, which have been biochemically characterized as having the function of regulating transcription (15).

Functional analysis using HaploReg v4.1. The effects of these SNPs on chromatin structure and allele-specific transcription factor binding were determined using HaploReg v4.1 (16). HaploReg is used to explore annotations of non-coding genomes in the results of GWAS or novel variant sets (17). Using Linkage disequilibrium information from the 1000 Genome Project, linked SNPs and indels in nine cell types can be visualized and their chromatin states and effects on regulatory motifs can be predicted (17).

Functional analysis using rSNPBase. rSNPBase is another tool for identifying potential regulatory genes, focusing on regulatory SNPs involving multiple regulatory types, including proximal, distal and post-transcriptional regulation $(18,19)$. rSNPBase helps researchers to select candidate SNPs for further genetic studies (especially for quantitative trait locus studies), to identify SNPs with specific phenotypes and to explore in-depth molecular mechanisms (20). Through searching, SNPs are annotated by referring to experimentally

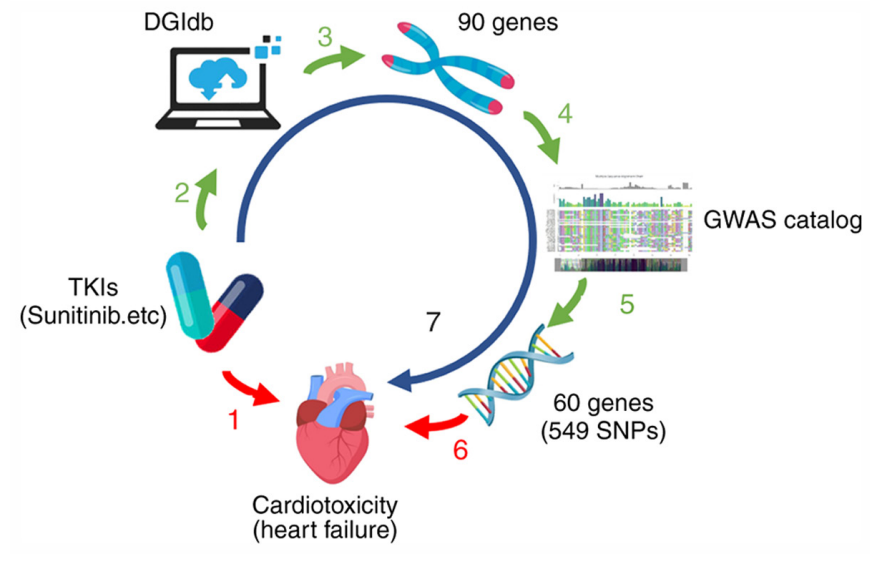

Figure 1. Experimental strategy. 1, TKIs raise the risk of HF. 2, All known TKI-targeting genes were extracted from the DGIdb. 3, DGIdb revealed 90 genes interacting with TKIs. 4, GWAS was performed between all common SNPs in chromosomal regions representing 90 genes and HF. 5, A total of 549 SNPs of 60 genes displayed significance for both TKI and HF risk. 6 , These genes were candidate risk genes for cardiotoxicity. 7, It can be hypothesized that TKI-induced genes associated with cardiotoxicity may be involved in adverse drug reactions. TKI, tyrosine kinase inhibitor; DGIdb, Drug Gene Interaction Database; SNP, single nucleotide polymorphism; HF, heart failure; GWAS, genome-wide association study.

supported regulatory elements (ENCODE data), encompassing a wide range of regulatory types (20).

Gene expression analysis of platelet-derived growth factor receptor (PDGFR) $\alpha$ in Genotype-Tissue Expression (GTEx) project. PDGFR $\alpha$ expression was assessed from the GTEx project using RNA sequencing (RNA-Seq) datasets (21-23). GTEx was designed based on data from 900 human donors from 53 sampling sites, including median gene expression levels in 51 tissues and two cell lines (24). The present study was based on data from 8,555 tissue samples obtained from 570 adult cadavers (25). Meanwhile, the UCSC Genome Browser was used to visualize interactions and expression between genomic regions (26).

Protein-protein interaction (PPI) network analysis of PDGFR a using the STRING database. Functional interactions between proteins can provide further insight on the molecular mechanisms of cellular processing. The present study constructed a PPI network of PDGFR $\alpha$ using the STRING database, which provides a key integration of PPIs, including known and predicted interactions. The STRING database aims to integrate and score all reported available sources of PPI information, and to complement computational predictions (27). Interacting pairs with high confidence (combined score $>0.7$ ) were selected to construct the PPI network.

\section{Results}

Selection of SNPs. The main approach used in the present study is illustrated in Fig. 1. A total of 90 genes that interacted with TKIs were identified in the DGIdb (Table SI) and these genes were studied in HF-associated GWAS datasets. A total of 549 common SNPs of 60 selected genes were chosen to represent the association between TKI molecular targets and cardiotoxicity $\left(\mathrm{P}<1 \times 10^{-5}\right)$. Subsequently, all genome-wide 


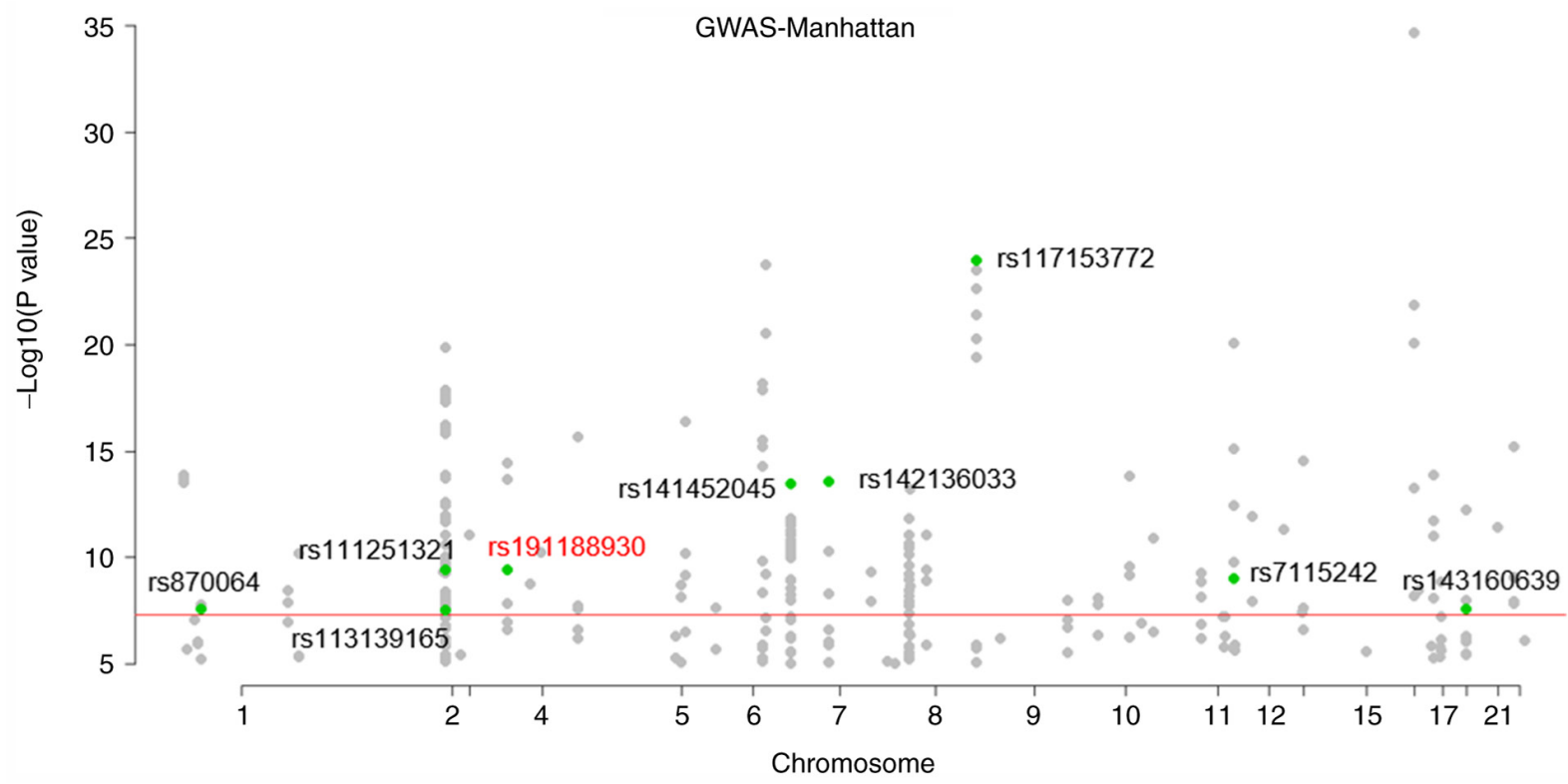

Figure 2. Aggregated Manhattan plot of the 549 SNPs from the GWAS results for association with cardiotoxicity and tyrosine kinase inhibitors targets. The red line indicates the level of genome-wide significance $\left(\mathrm{P}=5 \times 10^{-8}\right)$. Highlighted points represent SNPs of RegulomeDB with annotation score $\leq 3$, and rs191188930 was highly significant. SNP, single nucleotide polymorphism; GWAS, genome-wide association studies.

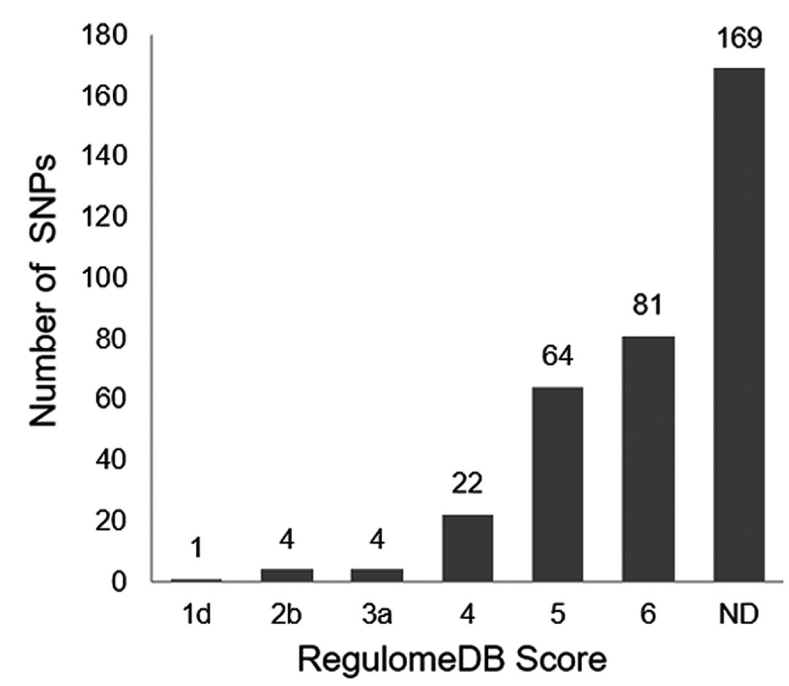

Figure 3. Number of SNPs ( $\mathrm{n}=345)$ in each RegulomeDB score. ND, no data; SNP, single nucleotide polymorphism.

association analysis was performed on these SNPs on the basis of the quantile-quantile plots. In total, 345 unique SNPs significantly associated with cardiotoxicity below the genome-wide significant threshold $\left(\mathrm{P}<5 \times 10^{-8}\right)$ are shown in the Manhattan plot in Fig. 2.

Regulatory analysis using RegulomeDB. In the RegulomeDB scoring system, the 345 SNPs selected from DGIdb were classified into six scores (scores 1-6), of which 176 SNPs had annotated scores ranging between 1 and 6 , and the remaining 169 SNPs had no annotated data (Fig. 3). Notably, the lower the score, the more likely the variant was located within the potential functional area (data not shown). Among the 345 SNPs, 9 possessed strong regulatory potential (score $\leq 3$; Table I). Among these 9 SNPs, rs191188930 on PDGFR $\alpha$ was associated with numerous TKI drugs (sunitinib, pazopanib, sorafenib, dasatinib and nilotinib; Table I), which will be the focus of the present systematic analysis.

Using histone modification analysis via RegulomeDB, rs191188930 was predicted to localize in enhancer histone markers (in adipose-derived mesenchymal stem cell cultured cells, IMR90 fetal lung fibroblasts cells, human endometrial stromal cells (hESC)-derived CD56 ${ }^{+}$mesoderm cultured cells, mesenchymal stem cell-derived adipocyte cultured cells, right atrium, foreskin fibroblast primary cells skin01, placenta, ovary, foreskin fibroblast primary cells skin02, fetal, muscle leg and foreskin keratinocyte primary cells skin03; Table II). The histone modification analysis of remaining SNPs is described in Table SII.

Functional analysis using HaploReg v4.1. Using HaploReg v4.1, rs191188930 was predicted to localize in enhancer histone markers (IMR90 fetal lung fibroblasts cells, foreskin fibroblast primary cells skin01, mesenchymal stem cell derived adipocyte cultured cells, adipose derived mesenchymal stem cells, foreskin fibroblast primary cells skin02, fetal muscle leg, placenta, foreskin fibroblast primary cells skin03, ovary and right atrium), DNase hypersensitivity (H1 derived mesenchymal stem cells, IMR90 fetal lung fibroblasts cell line, psoas muscles, foreskin fibroblast primary cells skin01, normal human epidermal keratinocyte primary cells, primary cells skin02, placenta, normal human adult dermal fibroblast primary cells and foreskin fibroblast), and motifs changed (Dmbx1 and GCNF) as described in Table III. The intronic annotation of rs191188930 indicated that it affected bound proteins such as CEBPB, but that there was no direct effect on promoter histone markers. More detailed results are shown in Table SIII.

Functional analysis using rSNPBase. Table IV integrates the results of rSNPBase, in which regulatory characteristics of SNPs retrieved for the four regulatory modes were 
Table I. List of prioritized putative regulatory single nucleotide polymorphisms.

\begin{tabular}{|c|c|c|c|c|c|c|c|c|}
\hline Variant & Chr & Position $^{\mathrm{a}}$ & $\mathrm{LD}\left(\mathrm{r}^{2}\right)$ & Ref & Alt & $\begin{array}{l}\text { RegulomeDB } \\
\text { score }^{b}\end{array}$ & Genes & Related tyrosine kinase inhibitors \\
\hline rs7115242 & 11 & 117037567 & 1 & G & A & $1 d$ & SIK3 & Dasatinib \\
\hline rs191188930 & 4 & 54256174 & 1 & G & $\mathrm{T}$ & $2 \mathrm{~b}$ & $\operatorname{PDGFR} \alpha$ & $\begin{array}{l}\text { Sunitinib, pazopanib, sorafenib, } \\
\text { dasatinib and nilotinib }\end{array}$ \\
\hline rs142136033 & 7 & 55100500 & 1 & G & A & $2 b$ & EGFR & $\begin{array}{l}\text { Sunitinib, sorafenib, dasatinib and } \\
\text { lapatinib }\end{array}$ \\
\hline rs143160639 & 17 & 78214341 & 1 & G & $\mathrm{T}$ & $2 \mathrm{~b}$ & BIRC5 & Lapatinib \\
\hline rs113139165 & 2 & 212520566 & 1 & $\mathrm{C}$ & $\mathrm{T}$ & $2 b$ & ERBB4 & Lapatinib \\
\hline rs870064 & 1 & 36487326 & 1 & $\mathrm{C}$ & $\mathrm{T}$ & $3 a$ & CSF3R & Dasatinib \\
\hline rs117153772 & 8 & 127908194 & 1 & G & $\mathrm{T}$ & $3 a$ & PVT1 & Imatinib mesylate \\
\hline rs141452045 & 6 & 152093883 & 1 & $\mathrm{C}$ & $\mathrm{T}$ & $3 a$ & ESR1 & Lapatinib \\
\hline rs111251321 & 2 & 212240009 & 1 & $\mathrm{C}$ & $\mathrm{T}$ & $3 a$ & ERBB4 & Lapatinib \\
\hline
\end{tabular}

aPosition (hg38). ${ }^{\mathrm{b} C u t-o f f, ~ R e g u l o m e D B ~ s c o r e ~} \leq 3$. Ref, reference allele; Alt, alternative allele; LD, linkage disequilibrium.

Table II. Key histone modification analysis of rs 191188930 using RegulomeDB.

\begin{tabular}{|c|c|c|c|}
\hline Method & Location & Chromatin function & Tissue/cells \\
\hline ChromHMM & chr4:55121000..55153200 & Genic enhancers & Adipose-derived mesenchymal stem cell cultured cells \\
\hline ChromHMM & chr4:55121000..55147600 & Strong transcription & $\begin{array}{l}\text { Human endometrial stromal cell-derived CD56 }{ }^{+} \\
\text {mesoderm cultured cells }\end{array}$ \\
\hline ChromHMM & chr4:55118600..55122400 & Genic enhancers & IMR90 fetal lung fibroblasts cells \\
\hline ChromHMM & chr4:55120800..55122400 & Enhancers & Mesenchymal stem cell-derived adipocyte cultured cells \\
\hline ChromHMM & chr4:55121200..55125400 & Genic enhancers & Foreskin fibroblast primary cells skin01 \\
\hline ChromHMM & chr4:55120000..55124400 & Enhancers & Placenta \\
\hline ChromHMM & chr4:55122200..55124800 & Enhancers & Ovary \\
\hline ChromHMM & chr4:55121200..55122400 & Enhancers & Foreskin fibroblast primary cells skin02 \\
\hline ChromHMM & chr4:55122000..55122400 & Enhancers & Foreskin keratinocyte primary cells skin03 \\
\hline ChromHMM & chr4:55122200..55122400 & Enhancers & Fetal muscle leg \\
\hline ChromHMM & chr4:55122200..55122400 & Enhancers & Right atrium \\
\hline
\end{tabular}

chr, chromosome; ChromHMM, chromatin hidden markov model.

assessed as 'Yes' or 'No'. rs7115242, rs143160639 and rs870064 were involved in proximal transcription regulation, which is associated with regulatory elements associated with DNA accessibility. rs7115242, rs143160639 and rs117153772 operated in distal regulation, which can modify chromatin interactions. Additionally, most SNPs interfered with the regulation of post-transcriptional RNA-binding proteins.

Functional analysis using PhenoScanner in GWAS. A total of 465 significant associations $(\mathrm{P}<0.01)$ were found using GWAS analysis in PhenoScanner. rs191188930 was significantly associated with other diseases or phenotypes other than cardiotoxicity, including acute pericarditis, aortic aneurysm and dissection, other necrotizing vasculopathies, urticaria, ascites and tubulo-interstitial nephritis (Table SIV).
Gene expression analysis of PDGFR $\alpha$ in GTEx. The highest median PDGFR $\alpha$ expression was found using GTEx datasets: Up to26.96readsperkilobasemillion(RPKM)in 'Cells-Transformed fibroblasts' (Ensembl gene ID, ENSG00000134853.7; genomic position, hg19 chr4:55095264-55148145) and the total median expression of all 53 tissues in the UCSC genome browser was 197.39 RPKM (Fig. 4).

PPI network analysis and functional pathway analysis of PDGFR $\alpha$ using the STRING database. Finally, the online STRING platform was used to perform PPI network analysis and examine protein interaction effects of PDGFR $\alpha$. The PPI network included a total of 11 nodes and 39 edges (PPI enrichment $\mathrm{P}<3.56 \times 10^{-7}$ ), as illustrated in Fig. 5. Based on previous research (4), three functional pathway genes (PDGFR $\alpha$, PDGFA and EGFR) affected by sunitinib were integrated (Fig. 6), which manifest downstream of the principle mechanism of sunitinib 
Table III. Functional analysis of related SNPs using HaploReg.

\begin{tabular}{|c|c|c|c|c|c|c|}
\hline Variant & $\begin{array}{c}\text { Promoter } \\
\text { histone markers }\end{array}$ & $\begin{array}{l}\text { Enhancer } \\
\text { histone } \\
\text { markers }\end{array}$ & $\begin{array}{c}\text { DNase } \\
\text { hyper- } \\
\text { sensitivity }\end{array}$ & $\begin{array}{l}\text { Bound } \\
\text { proteins }\end{array}$ & $\begin{array}{l}\text { Motifs } \\
\text { changed }\end{array}$ & $\begin{array}{l}\mathrm{dbSNP} \\
\text { functional } \\
\text { annotation }\end{array}$ \\
\hline rs7115242 & BLD, GI, BONE & 20 tissues & 21 tissues & KAP1 & TBX5 & Intronic \\
\hline rs191188930 & & 7 tissues & 8 tissues & CEBPB & Dmbx1, GCNF & Intronic \\
\hline rs142136033 & 4 tissues & 19 tissues & 14 tissues & POLR2A & $\begin{array}{l}\text { Ets, FEV, HMG-IY, } \\
\text { IK-3, STAT }\end{array}$ & Intronic \\
\hline rs143160639 & 23 tissues & 6 tissues & 19 tissues & $\begin{array}{l}28 \text { bound } \\
\text { proteins }\end{array}$ & $\begin{array}{l}\text { BCL, BDP1, CTCFL, } \\
\text { EWSR1-FLI1, Ets, Hic1, } \\
\text { INSM1, Myc, NERF1a, } \\
\text { Rad21, STAT }\end{array}$ & Missense \\
\hline rs113139165 & & HRT & 13 tissues & $\begin{array}{l}\text { CTCF, RAD21, } \\
\text { SMC3 }\end{array}$ & $\begin{array}{l}\text { Arnt, BAF155, Mrg, Mxi1, } \\
\text { Myc, Pou2f2, Tgif1, Znf143 }\end{array}$ & Intronic \\
\hline rs870064 & BLD, CRVX & 9 tissues & 11 tissues & $\begin{array}{l}\text { AP2ALPHA, } \\
\text { AP2GAMMA, } \\
\text { BAF155, HAE2F1 }\end{array}$ & PPAR, RXRA & Upstream \\
\hline rs 141452045 & & ESDR, FAT, & $\begin{array}{l}6 \text { tissues } \\
\text { SKIN }\end{array}$ & $\begin{array}{l}\text { SETDB } 1 \\
\text { TRIM28 }\end{array}$ & $\mathrm{EBF}$ & Intronic \\
\hline rs117153772 & 5 tissues & 16 tissues & 23 tissues & CJUN & & Intronic \\
\hline rs111251321 & & & & & $\begin{array}{l}\text { Gm397, Pou2f2, } \\
\text { Pou3f2, Pou3f3 }\end{array}$ & Intronic \\
\hline
\end{tabular}

dbSNP, database of single nucleotide polymorphism; BLD, GI, BONE: Primary T cells from cord blood, primary hematopoietic stem cells G-CSF-mobilized male, duodenum mucosa, osteoblast primary cells; BLD, CRVX: Primary neutrophils from peripheral blood, HeLa-S3 cervical cells, monocytes-CD14 ${ }^{+}$R01746 primary cells; HRT, Fetal heart; ESDR, FAT, SKIN: H1 BMP4-derived trophoblast cultured cells, adipose-derived mesenchymal stem cell cultured cells, foreskin fibroblast primary cells skin01, foreskin fibroblast primary cells skin02.

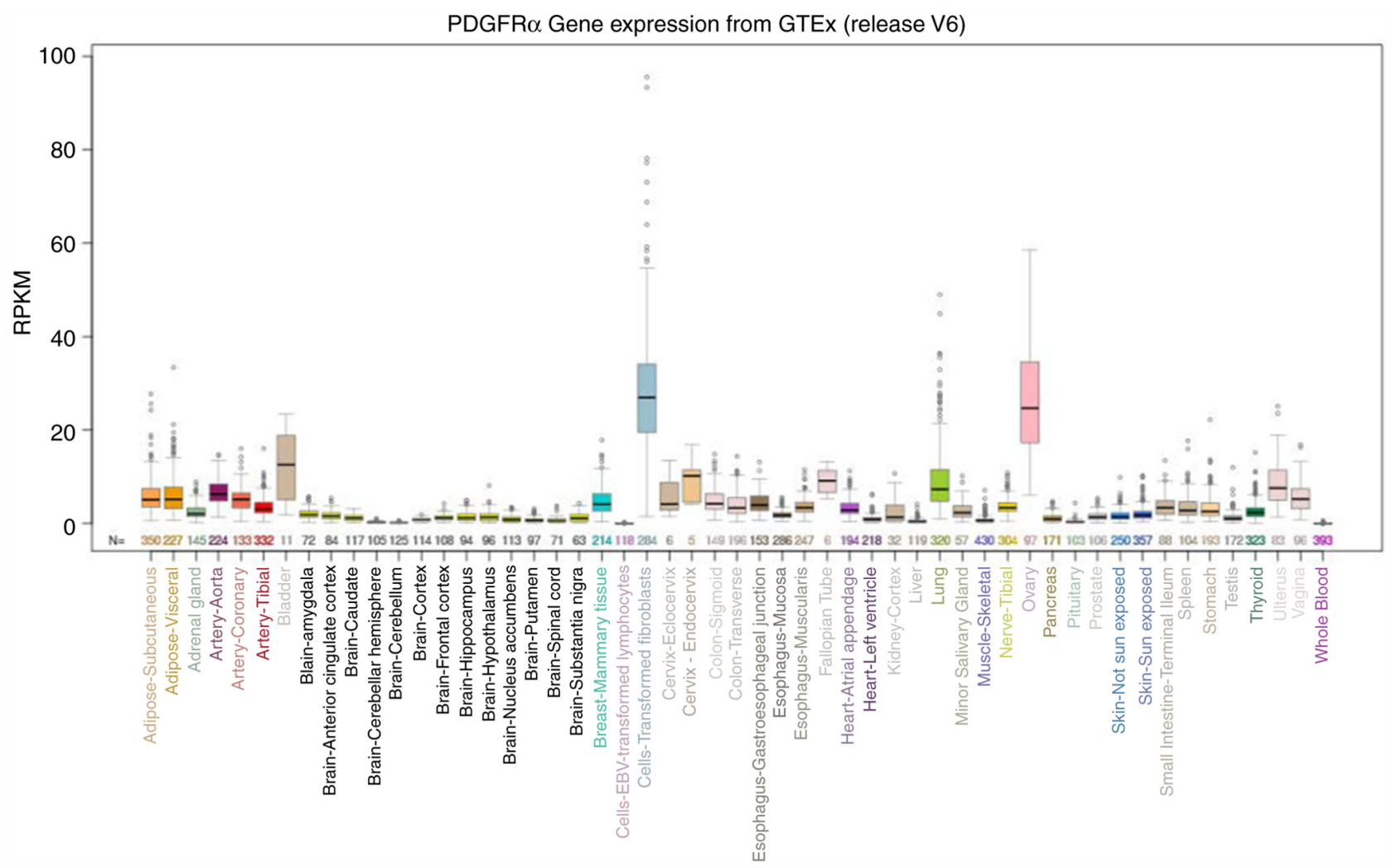

Figure 4. Gene expression levels of PDGFR $\alpha$ from GTEx using the University of California Santa Cruz Genome Browser. PDGFR, platelet-derived growth factor receptor; GTEx, Genotype-Tissue Expression; RPKM, reads per kilobase million. 
Table IV. Regulatory features of retrieved SNPs in four regulation manners using rSNPBase.

\begin{tabular}{lccccccc}
\hline SNP_ID & rSNP & $\begin{array}{c}\text { LD-proxy of } \\
\text { rSNP (r2>0.8) }\end{array}$ & $\begin{array}{c}\text { Proximal } \\
\text { regulation }\end{array}$ & $\begin{array}{c}\text { Distal } \\
\text { regulation }\end{array}$ & $\begin{array}{c}\text { microRNA } \\
\text { regulation }\end{array}$ & $\begin{array}{c}\text { RNA binding protein } \\
\text { mediated regulation }\end{array}$ & eQTL \\
\hline rs7115242 & Yes & Yes & Yes & Yes & No & Yes & Yes \\
rs191188930 & Yes & No & No & No & No & Yes & No \\
rs142136033 & No & No & No & No & No & No & No \\
rs143160639 & Yes & No & Yes & Yes & No & Yes & No \\
rs113139165 & Yes & No & No & No & No & Yes & No \\
rs870064 & Yes & Yes & Yes & No & No & No & No \\
rs141452045 & Yes & No & No & No & No & Yes & No \\
rs117153772 & Yes & No & No & Yes & No & No & No \\
rs111251321 & Yes & No & No & No & No & Yes & No \\
\hline
\end{tabular}

rSNP, regulatory single nucleotide polymorphism; LD, Linkage disequilibrium; eQTL, expression quantitative trait loci.

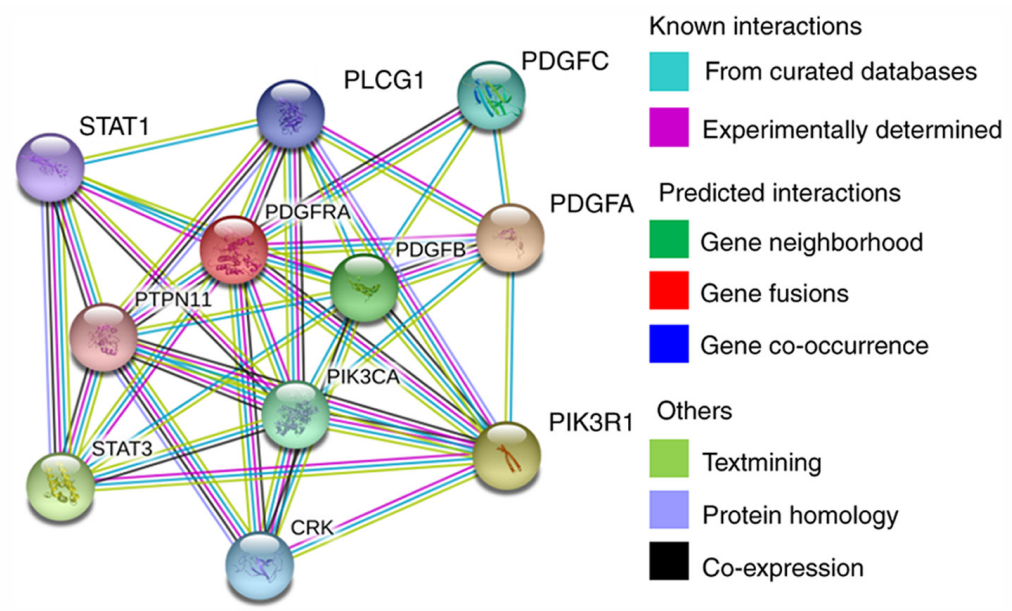

Figure 5. Protein-protein interaction network of PDGFR $\alpha$ using the Search Tool for the Retrieval of Interacting Genes/Proteins database.PDGFR, platelet-derived growth factor receptor.

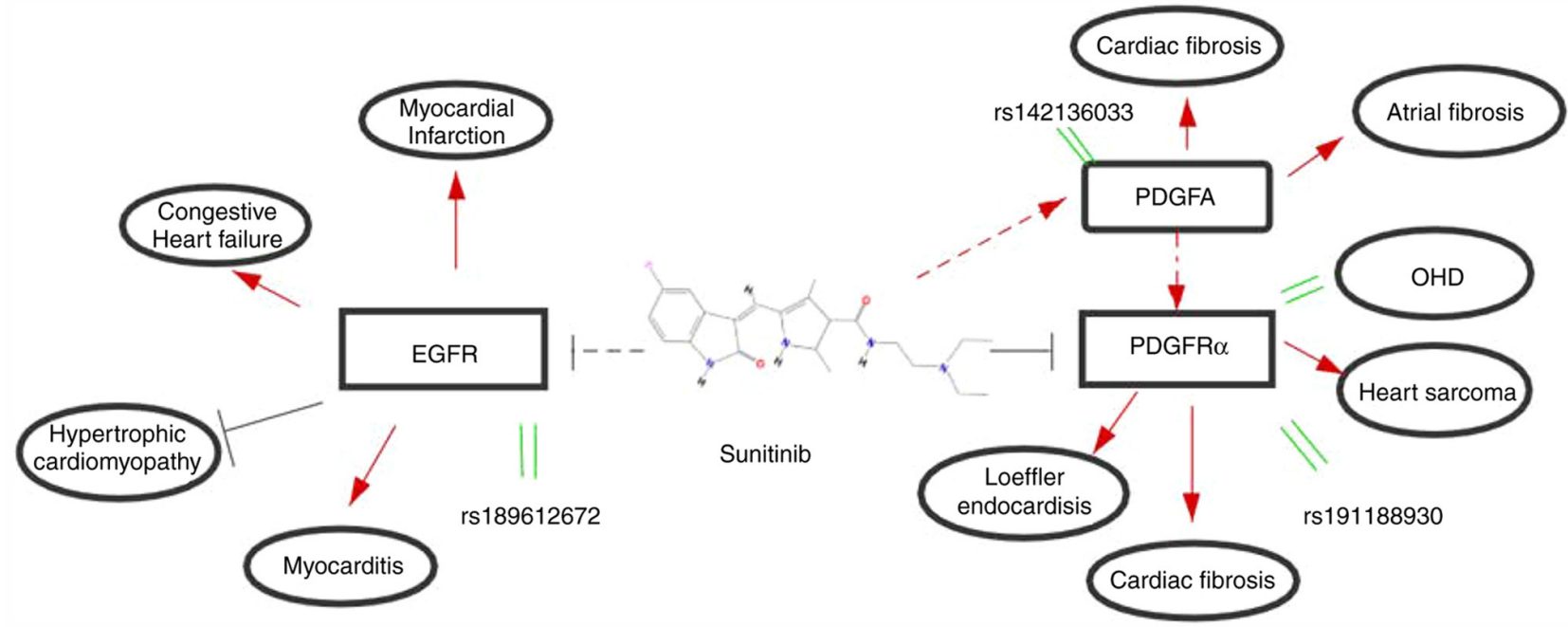

Figure 6. Pharmacodynamic effects of sunitinib treatment and genetically associated signals. All SNPs and their functional implications are based on cardiotoxicity risk alleles. The rs numbers indicate the lead SNPs and their hypothesized function. T-shaped lines indicate inhibition, parallel green lines indicate an unknown effect and red arrows indicate induction. Dotted lines indicate a potential intermediate functional link. Potential associations were reported for EGFR with myocardial infarction, congestive heart failure, hypertrophic cardiomyopathy and myocarditis. PDGFA was strongly associated with cardiac fibrosis and atrial fibrosis. PDGFR $\alpha$ was strongly associated with cardiac fibrosis, Loeffler endocarditis, heart sarcoma and OHD. The function of SNPs has not been reported. EGFR, epidermal growth factor receptor; PDGFA, platelet-derived growth factor subunit A; PDGFR $\alpha$, platelet-derived growth factor receptor $\alpha$; OHD, organic heart disease. 
inhibition. Potential associations were reported for EGFR with myocardial infarction, congestive heart failure, hypertrophic cardiomyopathy and myocarditis. PDGFA was strongly associated with cardiac fibrosis and atrial fibrosis. PDGFR $\alpha$ was strongly associated with cardiac fibrosis, Loeffler endocarditis, heart sarcoma and organic heart disease.

\section{Discussion}

The present study comprehensively investigated the possible functional relevance of the molecular targets of TKIs associated with cardiotoxicity by performing integrative analyses of publicly available datasets. The DGIdb listed 90 genes what interacted with TKIs, a type of pharmaceutical drugs that inhibit tyrosine kinases by competitively binding to and inhibiting their ATP binding pocket (4). TKIs are characterized by being multi-targeted anticancer agents that lack sufficient specificity, which markedly increases the risk of cardiotoxicity (5).

Over the last decade, GWAS has greatly advanced the understanding of the genetic basis of HF. To identify genes associated with TKIs, $549 \mathrm{HF}$-associated SNPs were identified from GWAS. However, not all GWAS-recorded SNPs serve a role in the pathogenesis of disease. Some SNPs are more likely to be functional in TKI-induced HF with significantly high annotation scores. Therefore, the present study performed comprehensive functional analyses of these SNPs utilizing multiple bioinformatics databases, including RegulomeDB, HaploReg v4.1, PhenoScanner v2, rSNPBase, GTEx and STRING, to prioritize and identify potential drug targets.

Using RegulomeDB, 9 SNPs possessed strong regulatory potential with a score $\leq 3$, and rs191188930 on PDGFR $\alpha$ (score 2b) was associated with five TKI drugs (sunitinib, pazopanib, sorafenib, dasatinib and nilotinib). Its annotation score (2b) suggested that it is more likely to affect binding (transcription factor binding), DNase footprint, DNase peak and any motif. Additionally, rs191188930 was predicted to localize in enhancer histone markers (adipose-derived mesenchymal stem cell cultured cells, IMR90 fetal lung fibroblasts cells, hESC-derived CD56 ${ }^{+}$mesoderm cultured cells, mesenchymal stem cell-derived adipocyte cultured cells, right atrium, foreskin fibroblast primary cells skin01, placenta, ovary, foreskin fibroblast primary cells skin02, fetal, muscle leg and foreskin keratinocyte primary cells skin03). Using HaploReg v4.1, rs191188930 was predicted to localize in enhancer histone markers (IMR90 fetal lung fibroblasts cells, foreskin fibroblast primary cells skin01, mesenchymal stem cell-derived adipocyte cultured cells, adipose-derived mesenchymal stem cells, foreskin fibroblast primary cells skin02, fetal muscle leg, placenta, foreskin fibroblast primary cells skin03, ovary and right atrium). Hence, the results obtained with HaploReg v4.1 were similar to those with RegulomeDB. Additionally, functional annotations by rSNPBase provided reliable evidence of the potential of rs191188930 in the susceptibility of TKI-induced cardiotoxicity. GWAS analysis in PhenoScanner revealed a total of 465 significant associations $(\mathrm{P}<0.01)$. Additionally, rs191188930 was significantly associated with other diseases or phenotypes other than cardiotoxicity, including acute pericarditis, aortic aneurysm and dissection, other necrotizing vasculopathies, urticaria, ascites and tubulo-interstitial nephritis.

PDGFs and their tyrosine kinase receptors are instrumental in adult organ diseases and embryonic organogenesis; the biological effects of PDGFs are produced by the activation of two tyrosine kinase receptors, PDGFR $\alpha$ and PDGFR $\beta(28,29)$. PDGFR $\alpha$ plays significant roles in embryonic organogenesis, differentiation, migration and function of specialized mesenchymal cells $(28,29)$. Particularly, PDGFR $\alpha$ is expressed by multifunctional cardiovascular progenitor cells in mouse and human embryonic stem cell systems (30).

Using 53 tissues from GTEx RNA-Seq data from 570 donors (8,555 samples), the highest median PDGFR $\alpha$ expression was identified: Up to 26.96 RPKM in 'Cells-Transformed fibroblasts'. Concurrently, PPI analysis of PDGFR $\alpha$ using the STRING database provided a direction for further research on the pathway of cardiotoxicity caused by TKIs.

The present study successfully prioritized SNPs and annotated the function of TKI-induced genes associated with HF, providing a foundation for further understanding of TKI-induced pathogenesis and the molecular mechanism of cardiotoxicity. The present findings indicated that rs191188930 was significantly associated with cardiotoxicity induced by TKIs. However, these computational prediction findings should be pragmatically handled and verified experimentally using appropriate systems before being considered for genomic medicine. Polymorphism studies should be followed up to assess the potential association of SNPs with more complex, clinical disease-related endpoints. Further research is required to elucidate the mechanisms of SNPs in their biological function.

\section{Acknowledgements}

Not applicable.

\section{Funding}

The present study was supported by the National Natural Science Foundation of China (grant nos. 81770255 and 82000381) and the Heilongjiang Province Postdoctoral Science Foundation (grant no. LBH-Z19188).

\section{Availability of data and materials}

The datasets generated and/or analyzed during the current study are available in the following repositories: DGIdb (http://dgidb. org/search_interactions), RegulomeDB (https://regulomedb. org/regulome-search/),HaploReg v4.1 (https://pubs.broadinstitute. org/mammals/haploreg/haploreg.php), rSNPBase, (http://rsnp. psych.ac.cn/), PhenoScanner (http://www.phenoscanner.medschl. cam.ac.uk/?tdsourcetag=s_pctim_aiomsg), GTEx (http://genome. ucsc.edu/) and STRING (https://string-db.org/cgi/network.pl?tas $\mathrm{kId}=\mathrm{xDOhbN0qKFY4 \& allnodes}=1)$.

\section{Authors' contributions}

YL and YZ conceived and designed the study. YL, WW and RG analyzed the data. YL and XX acquired the data. YL, RG and XX contributed to the writing of the manuscript. All authors read and approved the final manuscript and agree to be 
accountable for all aspects of the research in ensuring that the accuracy or integrity of any part of the work are appropriately investigated and resolved.

\section{Ethics approval and consent to participate}

Not applicable.

\section{Patient consent for publication}

Not applicable.

\section{Competing interests}

The authors declare that they have no competing interests.

\section{References}

1. Zamorano JL, Lancellotti P, Rodriguez Munoz D, Aboyans V, Asteggiano R, Galderisi M, Habib G, Lenihan DJ, Lip GYH, Lyon AR, et al: 2016 ESC position paper on cancer treatments and cardiovascular toxicity developed under the auspices of the ESC committee for practice guidelines: The task force for cancer treatments and cardiovascular toxicity of the European society of cardiology (ESC). Eur Heart J 37: 2768-2801, 2016.

2. Gyongyosi M, Lukovic D, Zlabinger K, Spannbauer A, Gugerell A, Pavo N, Traxler D, Pils D, Maurer G, Jakab A, et al: Liposomal doxorubicin attenuates cardiotoxicity via induction of interferon-related DNA damage resistance. Cardiovasc Res 116 : 970-982, 2020

3. Gupta SK, Garg A, Bar C, Chatterjee S, Foinquinos A, Milting H, Streckfuß-Bömeke K, Fiedler J and Thum T: Quaking inhibits doxorubicin-mediated cardiotoxicity through regulation of cardiac circular RNA expression. Circ Res 122: 246-254, 2018

4. Force T, Krause DS and Van Etten RA: Molecular mechanisms of cardiotoxicity of tyrosine kinase inhibition. Nat Rev Cancer 7 : 332-344, 2007.

5. Skubitz KM: Cardiotoxicity monitoring in patients treated with tyrosine kinase inhibitors. Oncologist 24: e600-e602, 2019.

6. Santoni M, Guerra F, Conti A, Lucarelli A, Rinaldi S, Belvederesi L, Capucci A and Berardi R: Incidence and risk of cardiotoxicity in cancer patients treated with targeted therapies. Cancer Treat Rev 59: 123-131, 2017.

7. Gill D, Georgakis MK, Koskeridis F, Jiang L, Feng Q, Wei WQ, Theodoratou E, Elliott P, Denny JC, Malik R, et al: Use of genetic variants related to antihypertensive drugs to inform on efficacy and side effects. Circulation 140: 270-279, 2019.

8. Griffith M, Griffith OL, Coffman AC, Weible JV, McMichael JF, Spies NC, Koval J, Das I, Callaway MB, Eldred JM, et al: DGIdb: Mining the druggable genome. Nat Methods 10: 1209-1210, 2013.

9. Wagner AH, Coffman AC, Ainscough BJ, Spies NC, Skidmore ZL, Campbell KM, Krysiak K, Pan D, McMichael JF, Eldred JM, et al: DGIdb 2.0: Mining clinically relevant drug-gene interactions. Nucleic Acids Res 44: D1036-D1044, 2016.

10. Cotto KC, Wagner AH, Feng YY, Kiwala S, Coffman AC, Spies G, Wollam A, Spies NC, Griffith OL and Griffith M: DGIdb 3.0: A redesign and expansion of the drug-gene interaction database. Nucleic Acids Res 46: D1068-D1073, 2018.

11. Kamat MA, Blackshaw JA, Young R, Surendran P, Burgess S, Danesh J, Butterworth AS and Staley JR: PhenoScanner V2: An expanded tool for searching human genotype-phenotype associations. Bioinformatics 35: 4851-4853, 2019.

12. Liao X, Lan C, Liao D, Tian J and Huang X: Exploration and detection of potential regulatory variants in refractive error GWAS. Sci Rep 6: 33090, 2016.

13. Boyle AP, Hong EL, Hariharan M, Cheng Y, Schaub MA, Kasowski M, Karczewski KJ, Park J, Hitz BC, Weng S, et al: Annotation of functional variation in personal genomes using RegulomeDB. Genome Res 22: 1790-1797, 2012.
14. Mesbah-Uddin M, Elango R, Banaganapalli B, Shaik NA and Al-Abbasi FA: In-silico analysis of inflammatory bowel disease (IBD) GWAS loci to novel connections. PLoS One 10: e0119420, 2015.

15. Wang E, Zhao H, Zhao D, Li L and Du L: Functional prediction of chronic kidney disease susceptibility gene PRKAG2 by comprehensively bioinformatics analysis. Front Genet 9: 573, 2018.

16. Ward LD and Kellis M: HaploReg v4: Systematic mining of putative causal variants, cell types, regulators and target genes for human complex traits and disease. Nucleic Acids Res 44: D877-D881, 2016.

17. Ward LD and Kellis M: HaploReg: A resource for exploring chromatin states, conservation, and regulatory motif alterations within sets of genetically linked variants. Nucleic Acids Res 40 (Database Issue): D930-D934, 2012.

18. Guo L, Du Y, Chang S, Zhang K and Wang J: rSNPBase: A database for curated regulatory SNPs. Nucleic Acids Res 42 (Database Issue): D1033-D1039, 2014.

19. Lou J, Gong J, Ke J, Tian J, Zhang Y, Li J, Yang Y, Zhu Y, Gong Y, Li L, et al: A functional polymorphism located at transcription factor binding sites, rs6695837 near LAMC1 gene, confers risk of colorectal cancer in Chinese populations. Carcinogenesis 38: 177-183, 2017.

20. Ganguly K, Saha T, Saha A, Dutta T, Banerjee S, Sengupta D, Bhattacharya S, Ghosh S and Sengupta M: Meta-analysis and prioritization of human skin pigmentation-associated GWAS-SNPs using ENCODE data-based web-tools. Arch Dermatol Res 311: 163-171, 2019.

21. Casper J, Zweig AS, Villarreal C, Tyner C, Speir ML, Rosenbloom KR, Raney BJ, Lee CM, Lee BT, Karolchik D, et al: The UCSC genome browser database: 2018 update. Nucleic Acids Res 46: D762-D769, 2018.

22. Haeussler M, Zweig AS, Tyner C, Speir ML, Rosenbloom KR, Raney BJ, Lee CM, Lee BT, Hinrichs AS, Gonzalez JN, et al: The UCSC genome browser database: 2019 update. Nucleic Acids Res 47: D853-D858, 2019.

23. Lee CM, Barber GP, Casper J, Clawson H, Diekhans M, Gonzalez JN, Hinrichs AS, Lee BT, Nassar LR, Powell CC, et al: UCSC genome browser enters 20th year. Nucleic Acids Res 48: D756-D761, 2020.

24. McCall MN, Illei PB and Halushka MK: Complex sources of variation in tissue expression data: Analysis of the GTEx lung transcriptome. Am J Hum Genet 99: 624-635, 2016.

25. Carithers LJ, Ardlie K, Barcus M, Branton PA, Britton A, Buia SA, Compton CC, DeLuca DS, Peter-Demchok J, Gelfand ET, et al: A novel approach to high-quality postmortem tissue procurement: The GTEx project. Biopreserv Biobank 13: 311-319, 2015.

26. Karolchik D, Barber GP, Casper J, Clawson H, Cline MS, Diekhans M, Dreszer TR, Fujita PA, Guruvadoo L, Haeussler M, et al: The UCSC genome browser database: 2014 update. Nucleic Acids Res 42: D764-D770, 2014.

27. Szklarczyk D, Gable AL, Lyon D, Junge A, Wyder S, Huerta-Cepas J, Simonovic M, Doncheva NT, Morris JH, Bork P, et al: STRING v11: Protein-protein association networks with increased coverage, supporting functional discovery in genome-wide experimental datasets. Nucleic Acids Res 47: D607-D613, 2019.

28. Tallquist MD and Soriano P: Cell autonomous requirement for PDGFRalpha in populations of cranial and cardiac neural crest cells. Development 130: 507-518, 2003.

29. Hoch RV and Soriano P: Roles of PDGF in animal development. Development 130: 4769-4784, 2003.

30. Hong SP, Song S, Cho SW, Lee S, Koh BI, Bae H, Kim KH, Park JS, Do HS, Im I, et al: Generation of PDGFR $\alpha^{+}$cardioblasts from pluripotent stem cells. Sci Rep 7: 41840, 2017.

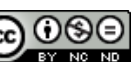

This work is licensed under a Creative Commons Attribution-NonCommercial-NoDerivatives 4.0 International (CC BY-NC-ND 4.0) License. 